\title{
RECENT ADVANCES IN THE THEORY OF NUCLEAR FORCES AND ITS IMPACT ON MICROSCOPIC NUCLEAR STRUCTURE*
}

\author{
R. Machleidt \\ Department of Physics, University of Idaho, \\ Moscow, Idaho 83844, U.S.A. \\ E-mail: machleid@uidaho.edu
}

\begin{abstract}
The theory of nuclear forces has made great progress since the turn of the millenium using the framework of chiral effective field theory (ChEFT). The advantage of this approach, which was originally proposed by Weinberg, is that it has a firm basis in quantumchromodynamics and allows for quantitative calculations. Moreover, this theory generates two-nucleon forces $(2 \mathrm{NF})$ and many-body forces on an equal footing and provides an explanation for the empirically known fact that $2 \mathrm{NF} \gg 3 \mathrm{NF} \gg 4 \mathrm{NF}$. I will present the recent advances in more detail and put them into historical context. In addition, I will also provide a critical evaluation of the progress made including a discussion of the limitations of the ChEFT approach.
\end{abstract}

Keywords: Nuclear forces, nucleon-nucleon interaction, effective field theory, chiral perturbation theory, nuclear matter.

\section{Introduction and historical perspective}

The theory of nuclear forces has a long history (cf. Table 1). Based upon the seminal idea by Yukawa, ${ }^{1}$ first field-theoretic attempts to derive the nucleon-nucleon (NN) interaction focused on pion-exchange. While the one-pion exchange turned out to be very useful in explaining $\mathrm{NN}$ scattering data and the properties of the deuteron, multi-pion exchange was beset with serious ambiguities. Thus, the "pion theories" of the 1950s are generally judged as failures - for reasons we understand today: pion dynamics is constrained by chiral symmetry, a crucial point that was unknown in the 1950s.

Historically, the experimental discovery of heavy mesons in the early 1960s saved the situation. The one-boson-exchange (OBE) model $^{2}$ emerged which is still the most economical and quantitative phenomenology for describing the nuclear force..$^{3,4}$ The weak point of this model, however, is the scalar-isoscalar "sigma" or "epsilon" boson, for which the empirical evidence remains controversial. Since this boson is associated with the correlated (or resonant) exchange of two pions, a vast theoretical effort that occupied more than a decade was launched to derive the $2 \pi$-exchange contribution to the nuclear force, which creates the intermediate range attraction.

*Talk presented at EXOCT 2007, Catania, Italy, June 11-15, 2007. 
Table 1. Seven Decades of Struggle: The Theory of Nuclear Forces

\begin{tabular}{|c|c|}
\hline 1935 & Yukawa: Meson Theory \\
\hline 1950's & $\begin{array}{c}\text { The "Pion Theories" } \\
\text { One-Pion Exchange: o.k. } \\
\text { Multi-Pion Exchange: disaster }\end{array}$ \\
\hline 1960's & $\begin{array}{c}\text { Many pions } \equiv \text { multi-pion resonances: } \\
\boldsymbol{\sigma}, \boldsymbol{\rho}, \boldsymbol{\omega}, \ldots \\
\text { The One-Boson-Exchange Model }\end{array}$ \\
\hline 1970's & $\begin{array}{c}\text { Refine meson theory: } \\
\text { Sophisticated } \mathbf{2} \boldsymbol{\pi} \text { exchange models } \\
\text { (Stony Brook, Paris, Bonn) }\end{array}$ \\
\hline 1980's & $\begin{array}{c}\text { Nuclear physicists discover } \\
\text { QCD } \\
\text { Quark Cluster Models }\end{array}$ \\
\hline $\begin{array}{l}\text { 1990's } \\
\text { and beyond }\end{array}$ & $\begin{array}{c}\text { Nuclear physicists discover EFT } \\
\text { Weinberg, van Kolck } \\
\text { Back to Meson Theory! } \\
\text { But, with Chiral Symmetry }\end{array}$ \\
\hline
\end{tabular}

For this, dispersion theory as well as field theory were invoked producing the Paris ${ }^{5}$ and the Bonn ${ }^{2,6}$ potentials.

The nuclear force problem appeared to be solved; however, with the discovery of quantum chromo-dynamics (QCD), all "meson theories" were relegated to models and the attempts to derive the nuclear force started all over again.

The problem with a derivation from QCD is that this theory is non-perturbative in the low-energy regime characteristic of nuclear physics, which makes direct solutions impossible. Therefore, during the first round of new attempts, QCD-inspired quark models ${ }^{7}$ became popular. These models are able to reproduce qualitatively and, in some cases, semi-quantitatively the gross features of the nuclear force. ${ }^{8,9}$ However, on a critical note, it has been pointed out that these quark-based approaches are nothing but another set of models and, thus, do not represent any fundamental progress. Equally well, one may then stay with the simpler and much more quantitative meson models.

A major breakthrough occurred when the concept of an effective field theory (EFT) was introduced and applied to low-energy QCD. ${ }^{10}$

Note that the QCD Lagrangian for massless up and down quarks is chirally symmetric, i. e., it is invariant under global flavor $S U(2)_{L} \times S U(2)_{R}$ equivalent to $S U(2)_{V} \times S U(2)_{A}$ (vector and axial vector) transformations. The axial symmetry is spontaneously broken as evidenced in the absence of parity doublets in the low-mass hadron spectrum. This implies the existence of three massless Goldstone bosons which are identified with the three pions $\left(\pi^{ \pm}, \pi^{0}\right)$. The non-zero, but small, pion mass is a consequence of the fact that the up and down quark masses are not exactly zero either (some small, but explicit symmetry breaking). Thus, we arrive at a lowenergy scenario that consists of pions and nucleons interacting via a force governed 
by spontaneously broken approximate chiral symmetry.

To create an effective field theory describing this scenario, one has to write down the most general Lagrangian consistent with the assumed symmetry principles, particularly the (broken) chiral symmetry of QCD. ${ }^{10}$ At low energy, the effective degrees of freedom are pions and nucleons rather than quarks and gluons; heavy mesons and nucleon resonances are "integrated out". So, the circle of history is closing and we are back to Yukawa's meson theory, except that we have learned to add one important refinement to the theory: broken chiral symmetry is a crucial constraint that generates and controls the dynamics and establishes a clear connection with the underlying theory, QCD.

It is the purpose of the remainder of this contribution to describe the EFT approach to nuclear forces in more detail.

\section{Chiral perturbation theory and the hierarchy of nuclear forces}

The chiral effective Lagrangian is given by an infinite series of terms with increasing number of derivatives and/or nucleon fields, with the dependence of each term on the pion field prescribed by the rules of broken chiral symmetry. Applying this Lagrangian to NN scattering generates an unlimited number of Feynman diagrams. However, Weinberg showed ${ }^{11}$ that a systematic expansion exists in terms of $\left(Q / \Lambda_{\chi}\right)^{\nu}$, where $Q$ denotes a momentum or pion mass, $\Lambda_{\chi} \approx 1 \mathrm{GeV}$ is the chiral symmetry breaking scale, and $\nu \geq 0$ (cf. Fig. 1). This has become known as chiral perturbation theory $(\chi \mathrm{PT})$. For a given order $\nu$, the number of terms is finite and calculable; these terms are uniquely defined and the prediction at each order is model-independent. By going to higher orders, the amplitude can be calculated to any desired accuracy.

Following the first initiative by Weinberg, ${ }^{11}$ pioneering work was performed by Ordóñez, Ray, and van Kolck ${ }^{12,13}$ who constructed a NN potential in coordinate space based upon $\chi \mathrm{PT}$ at next-to-next-to-leading order (NNLO; $\nu=3$ ). The results were encouraging and many researchers became attracted to the new field. Kaiser, Brockmann, and Weise ${ }^{14}$ presented the first model-independent prediction for the NN amplitudes of peripheral partial waves at NNLO. Epelbaum et al. ${ }^{15}$ developed the first momentum-space NN potential at NNLO, and Entem and Machleidt ${ }^{16}$ presented the first potential at $\mathrm{N}^{3} \mathrm{LO}(\nu=4)$.

In $\chi \mathrm{PT}$, the $\mathrm{NN}$ amplitude is uniquely determined by two classes of contributions: contact terms and pion-exchange diagrams. There are two contacts of order $Q^{0}\left[\mathcal{O}\left(Q^{0}\right)\right]$ represented by the four-nucleon graph with a small-dot vertex shown in the first row of Fig. 1. The corresponding graph in the second row, four nucleon legs and a solid square, represent the seven contact terms of $\mathcal{O}\left(Q^{2}\right)$. Finally, at $\mathcal{O}\left(Q^{4}\right)$, we have 15 contact contributions represented by a four-nucleon graph with a solid diamond.

Now, turning to the pion contributions: At leading order $\left[\mathrm{LO}, \mathcal{O}\left(Q^{0}\right), \nu=0\right]$, there is only the wellknown static one-pion exchange, second diagram in the first row 

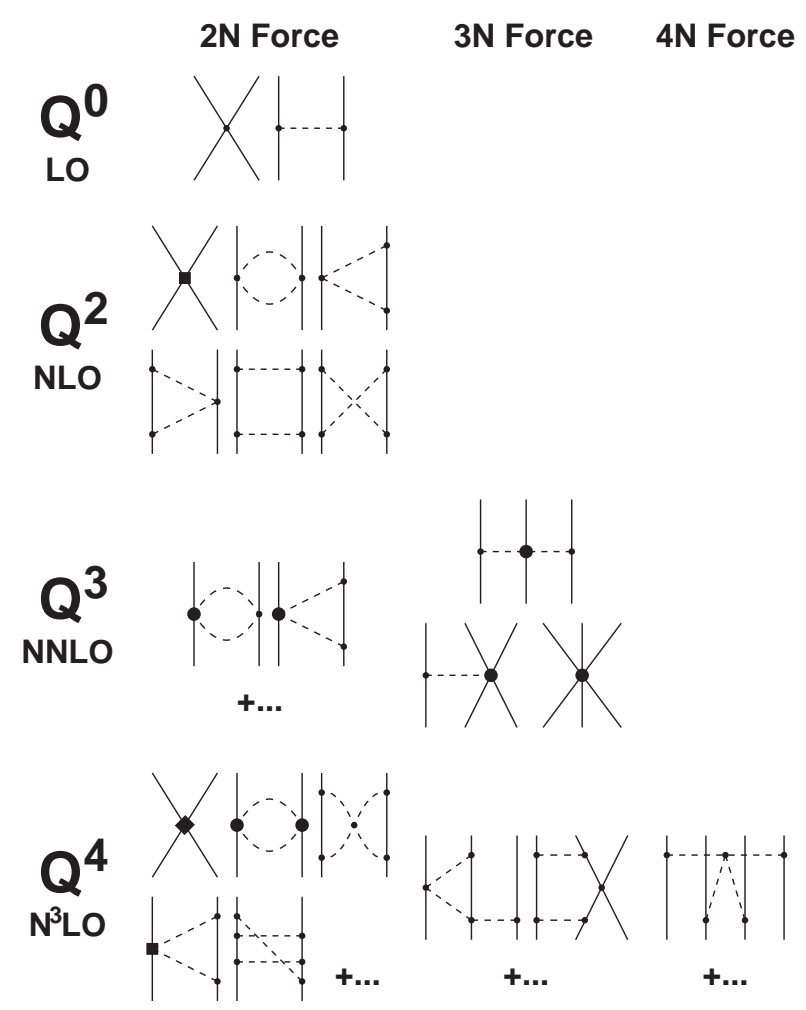

Fig. 1. Hierarchy of nuclear forces in $\chi \mathrm{PT}$. Solid lines represent nucleons and dashed lines pions. Further explanations are given in the text.

of Fig. 1. Two-pion exchange (TPE) starts at next-to-leading order (NLO, $\nu=2)$ and all diagrams of this leading-order two-pion exchange are shown. Further TPE contributions occur in any higher order. Of this sub-leading TPE, we show only two representative diagrams at NNLO and three diagrams at $\mathrm{N}^{3} \mathrm{LO}$. The TPE at $\mathrm{N}^{3} \mathrm{LO}$ has been calculated first by Kaiser. ${ }^{17}$ All $2 \pi$ exchange diagrams/contributions up to $\mathrm{N}^{3} \mathrm{LO}$ are summarized in a pedagogical and systematic fashion in Ref. 18 where the model-independent results for $\mathrm{NN}$ scattering in peripheral partial waves are also shown.

Finally, there is also three-pion exchange, which shows up for the first time at $\mathrm{N}^{3} \mathrm{LO}$ (two loops; one representative $3 \pi$ diagram is included in Fig. 1 ). In Ref. 19 it was demonstrated that the $3 \pi$ contribution at this order is negligible.

One important advantage of $\chi \mathrm{PT}$ is that it makes specific predictions also for many-body forces. For a given order of $\chi \mathrm{PT}$, two-nucleon forces $(2 \mathrm{NF})$, threenucleon forces $(3 \mathrm{NF}), \ldots$ are generated on the same footing (cf. Fig. 1). At LO, there are no $3 \mathrm{NF}$, and at next-to-leading order (NLO), all 3NF terms cancel. ${ }^{11,20}$ However, at NNLO and higher orders, well-defined, nonvanishing $3 \mathrm{NF}$ occur. ${ }^{20,21}$ Since 3NF show up for the first time at NNLO, they are weak. Four-nucleon forces 
Table 2. $\chi^{2}$ /datum for the reproduction of the $1999 n p$ database by families of $n p$ potentials at NLO and NNLO constructed by the Bochum/Juelich group. ${ }^{22}$

\begin{tabular}{cccc}
\hline \hline & \# of $n p$ & NLO & Bochum/Juelich \\
Bin $(\mathrm{MeV})$ & data & $4-5$ & NNLO \\
\hline \hline $0-100$ & 1058 & $77-121$ & $1.4-1.9$ \\
$100-190$ & 501 & $140-220$ & $12-32$ \\
$190-290$ & 843 & $67-105$ & $25-69$ \\
\hline $0-290$ & 2402 & $12-27$ \\
\hline \hline
\end{tabular}

(4NF) occur first at $\mathrm{N}^{3} \mathrm{LO}$ and, therefore, they are even weaker.

\section{Chiral NN potentials}

The two-nucleon system is non-perturbative as evidenced by the presence of shallow bound states and large scattering lengths. Weinberg ${ }^{11}$ showed that the strong enhancement of the scattering amplitude arises from purely nucleonic intermediate states. He therefore suggested to use perturbation theory to calculate the NN potential (Fig. 1) and to apply this potential in a scattering equation (LippmannSchwinger or Schrödinger equation) to obtain the NN amplitude. We follow this philosophy.

Chiral perturbation theory is a low-momentum expansion. It is valid only for momenta $Q \ll \Lambda_{\chi} \approx 1 \mathrm{GeV}$. Therefore, when a potential is constructed, all expressions (contacts and irreducible pion exchanges) are multiplied with a regulator function,

$$
\exp \left[-\left(\frac{p}{\Lambda}\right)^{2 n}-\left(\frac{p^{\prime}}{\Lambda}\right)^{2 n}\right]
$$

where $p$ and $p^{\prime}$ denote, respectively, the magnitudes of the initial and final nucleon momenta in the center-of-mass system (CMS); and $\Lambda \ll \Lambda_{\chi}$. The exponent $2 n$ is to be chosen such that the regulator generates powers which are beyond the order at which the calculation is conducted.

To what order in $\chi \mathrm{PT}$ do we have to go for sufficient accuracy? To discuss this issue on firm grounds, I show in Table 2 the $\chi^{2} /$ datum for the fit of the world $n p$ data below $290 \mathrm{MeV}$ for a family of $n p$ potentials at NLO and NNLO. The NLO potentials produce the horrendous $\chi^{2} /$ datum between 67 and 105, and the NNLO are between 12 and 27 . The rate of improvement from one order to the other is very impressive, but the quality of the reproduction of the $n p$ data at NLO and NNLO is obviously totally insufficient for reliable predictions.

Based upon these facts, it has been pointed out in 2002 by Entem and Machleidt ${ }^{18,23}$ that NNLO is insufficient and one has to proceed to $\mathrm{N}^{3} \mathrm{LO}$. Consequently, the first $\mathrm{N}^{3} \mathrm{LO}$ potential was created in $2003,{ }^{16}$ which showed that at this order a $\chi^{2} /$ datum comparable to the high-precision Argonne $V_{18}$ potential can, indeed, be achieved, see Tables 3 and 4 . This "Idaho" $\mathrm{N}^{3} \mathrm{LO}$ potential ${ }^{16}$ produces a $\chi^{2} /$ datum $=1.1$ for the world $n p$ data below $290 \mathrm{MeV}$ which compares well with the $\chi^{2} /$ datum 
Table 3. $\chi^{2} /$ datum for the reproduction of the $1999 \boldsymbol{n p}$ database by various $n p$ potentials. Numbers in parentheses denote cutoff parameters in units of $\mathrm{MeV}$.

\begin{tabular}{cc|c|c|c}
\hline \hline Bin $(\mathrm{MeV})$ & $\begin{array}{c}\text { Idaho } \\
\text { of } \boldsymbol{n p}\end{array}$ & $\begin{array}{c}\mathrm{N}^{3} \mathrm{LO}^{16} \\
(500-600)\end{array}$ & $\begin{array}{c}\text { Bochum/Juelich } \\
\mathrm{N}^{3} \mathrm{LO}^{25} \\
(600 / 700-450 / 500)\end{array}$ & $\begin{array}{c}\text { Argonne } \\
V_{18}{ }^{24}\end{array}$ \\
\hline \hline $0-100$ & data & $1.0-1.1$ & $1.0-1.1$ & 0.95 \\
$100-190$ & 1058 & $1.1-1.2$ & $1.3-1.8$ & 1.10 \\
$190-290$ & 501 & $1.2-1.4$ & $2.8-20.0$ & 1.11 \\
\hline $0-290$ & 843 & $1.1-1.3$ & $1.7-7.9$ & 1.04 \\
\hline \hline
\end{tabular}

$=1.04$ by the Argonne potential (Table 3). In 2005, also the Bochum/Juelich group produced several $\mathrm{N}^{3} \mathrm{LO} \mathrm{NN}$ potentials ${ }^{25}$ the best of which fits the $n p$ data with a $\chi^{2} /$ datum $=1.7$ and the worse with a $\chi^{2} /$ datum $=7.9$ (cf. Table 3 ). While 7.9 is clearly unacceptable for any meaningful application, a $\chi^{2} /$ datum of 1.7 may be sufficient for most purposes.

I turn now to the $p p$ data, Table 4 . Typically, $\chi^{2}$ for $p p$ data are larger than for $n p$ because of the higher precision of $p p$ data. Thus, the Argonne $V_{18}$ produces a $\chi^{2} /$ datum $=1.4$ for the world $p p$ data below $290 \mathrm{MeV}$ and the best Idaho $\mathrm{N}^{3} \mathrm{LO}$ $p p$ potential obtains 1.5. The fit by the best Bochum/Juelich $\mathrm{N}^{3} \mathrm{LO} p p$ potential results in a $\chi^{2} /$ datum $=2.9$ and the worst is 22.3 .

\section{Limitations of $\chi \mathbf{P T}$}

Since $\chi \mathrm{PT}$ is a low-momentum expansion, we have to expect limitations concerning its applicability. This is demonstrated in Fig. 2, where phase shift predictions by various NN potentials are shown up to $1000 \mathrm{MeV}$ lab. energy for the incident nucleon. The figure includes one representative of the family of the high-precision NN potentials, namely, the CD-Bonn potential ${ }^{4}$ (solid line), which obviously predicts the phase shifts correctly up to the highest energies shown, even though it was adjusted only up to $350 \mathrm{MeV}$. The same is true for other high-precision potentials, like Argonne $V_{18}{ }^{24}$ and the Nijmegen potentials. ${ }^{3}$ On the other hand, the chiral NN potentials at order $\mathrm{N}^{3} \mathrm{LO}$ (dashed line Ref. 16 and dotted line Ref. 25) do not make any reasonable predictions beyond about $300 \mathrm{MeV}$ lab. energy. This is, of course, not unexpected since $\chi \mathrm{PT}$ applies only for momenta $Q \ll \Lambda_{\chi} \approx 1 \mathrm{GeV}$, which is enforced by the regulator Eq. (1) where a typical choice for the cutoff param-

Table 4. $\chi^{2} /$ datum for the reproduction of the $1999 \boldsymbol{p p}$ database by various $p p$ potentials. Numbers in parentheses denote cutoff parameters in units of $\mathrm{MeV}$.

\begin{tabular}{cc|c|c|c}
\hline \hline Bin $(\mathrm{MeV})$ & $\begin{array}{c}\text { \# of } \boldsymbol{p} \boldsymbol{p} \\
\text { data }\end{array}$ & $\begin{array}{c}\text { Idaho } \\
\mathrm{N}^{3} \mathrm{LO}^{16} \\
(500-600)\end{array}$ & $\begin{array}{c}\text { Bochum/Juelich } \\
\mathrm{N}^{3} \mathrm{LO}^{25} \\
(600 / 700-450 / 500)\end{array}$ & $\begin{array}{c}\text { Argonne } \\
V_{18}{ }^{24}\end{array}$ \\
\hline \hline $0-100$ & 795 & $1.0-1.7$ & $1.0-3.8$ & 1.0 \\
$100-190$ & 411 & $1.5-1.9$ & $3.5-11.6$ & 1.3 \\
$190-290$ & 851 & $1.9-2.7$ & $4.3-44.4$ & 1.8 \\
\hline $0-290$ & 2057 & $1.5-2.1$ & $2.9-22.3$ & 1.4 \\
\hline \hline
\end{tabular}



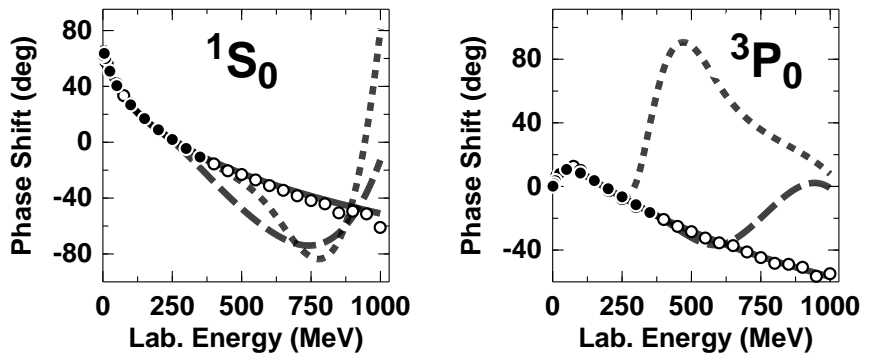

Fig. 2. $n p$ phase shifts of the ${ }^{1} S_{0}$ and ${ }^{3} P_{0}$ partial waves for lab. energies up to $1000 \mathrm{MeV}$. The solid curve shows the phase shifts predicted by the CD-Bonn potential. ${ }^{4}$ Note that this curve is hardly visible because it agrees with the data and, thus, is buried under the symbols representing the data. The dashed and the dotted lines are the predictions by the $\mathrm{N}^{3} \mathrm{LO}$ chiral potentials constructed by the Idaho ${ }^{16}$ and the Bochum/Juelich ${ }^{25}$ groups, respectively. Solid dots represent the Nijmegen multienergy $n p$ phase shift analysis ${ }^{26}$ and open circles the GWU/VPI single-energy $n p$ analysis SM99. ${ }^{27}$

eter is $\Lambda \approx 500 \mathrm{MeV}$. Thus, chiral potentials are reliable only for CMS momenta $p, p^{\prime} \lesssim 2.2 \mathrm{fm}^{-1}$. A Fermi momentum $k_{F} \approx 2.2 \mathrm{fm}^{-1}$ is equivalent to a nuclear matter density $\rho \approx 4 \rho_{0}$ where $\rho_{0}$ denotes nuclear matter saturation density. Nuclear matter calculations in which chiral two-nucleon potentials are applied can be found in Refs. 28,29. Note, however, that for "complete" calculations also the chiral 3NF must be included. In any case, based upon the above arguments, one may trust the $\chi \mathrm{PT}$ approach up to densities around $4 \rho_{0}$. In contrast, relativistic meson theory can be trusted to very high momenta (cf. the CD-Bonn curve in Fig. 2) and densities equivalent to those high momenta.

\section{Conclusions}

The theory of nuclear forces has made great strides since the turn of the millennium. Nucleon-nucleon potentials have been developed that are based on proper theory (EFT for low-energy QCD) and are of high-precision, at the same time. Moreover, the theory generates two- and many-body forces on an equal footing and provides a theoretical explanation for the empirically known fact that $2 \mathrm{NF} \gg 3 \mathrm{NF} \gg 4 \mathrm{NF}$.

At $\mathrm{N}^{3} \mathrm{LO},{ }^{16,18}$ the accuracy can be achieved that is necessary and sufficient for microscopic nuclear structure. First calculations applying the $\mathrm{N}^{3} \mathrm{LO}$ NN potential $^{16}$ in the (no-core) shell model, ${ }^{30-33}$ the coupled cluster formalism, ${ }^{34-38}$ and the unitary-model-operator approach ${ }^{39}$ have produced promising results.

The $3 \mathrm{NF}$ at NNLO is known ${ }^{20,21}$ and has been applied in few-nucleon reactions $^{21,40,41}$ as well as the structure of light nuclei. ${ }^{42-44}$ However, the famous ' $A_{y}$ puzzle' of nucleon-deuteron scattering is not resolved by the $3 \mathrm{NF}$ at NNLO. Thus, the most important outstanding issue is the $3 \mathrm{NF}$ at $\mathrm{N}^{3} \mathrm{LO}$, which is under construction. 


\section{Acknowledgments}

This work was supported in part by the U.S. National Science Foundation under Grant No. PHY-0099444.

\section{References}

1. H. Yukawa, Proc. Phys. Math. Soc. Japan 17, 48 (1935).

2. R. Machleidt, Adv. Nucl. Phys. 19, 189 (1989).

3. V.G.J. Stoks et al., Phys. Rev. C 49, 2950 (1994).

4. R. Machleidt, Phys. Rev. C 63, 024001 (2001).

5. M. Lacombe et al., Phys. Rev. C 21, 861 (1980).

6. R. Machleidt et al., Phys. Rep. 149, 1 (1987).

7. F. Myhrer et al., Rev. Mod. Phys. 60, 629 (1988).

8. D.R. Entem, F. Fernandez, and A. Valcarce, Phys. Rev. C 62, 034002 (2000).

9. G.H. Wu et al., Nucl. Phys. A673, 273 (2000).

10. S. Weinberg, Physica 96A, 327 (1979).

11. S. Weinberg, Nucl. Phys. B363, 3 (1991).

12. C. Ordóñez, L. Ray, and U. van Kolck, Phys. Rev. C 53, 2086 (1996).

13. U. van Kolck, Prog. Part. Nucl. Phys. 43, 337 (1999).

14. N. Kaiser et al., Nucl. Phys. A625, 758 (1997).

15. E. Epelbaum et al., Nucl. Phys. A671, 295 (2000).

16. D.R. Entem and R. Machleidt, Phys. Rev. C 68, 041001 (2003).

17. N. Kaiser, Phys. Rev. C 64, 057001 (2001); 65, 017001 (2002).

18. D. R. Entem and R. Machleidt, Phys. Rev. C 66, 014002 (2002).

19. N. Kaiser, Phys. Rev. C 61, 014003 (1999); ibid. 62, 024001 (2000).

20. U. van Kolck, Phys. Rev. C 49, 2932 (1994).

21. E. Epelbaum et al., Phys. Rev. C 66, 064001 (2002).

22. E. Epelbaum, W. Glöckle, and U.-G. Meißner, Eur. Phys. J. A19, 401 (2004).

23. D. R. Entem and R. Machleidt, Phys. Lett. B 524, 93 (2002).

24. R.B. Wiringa, V.G.J. Stoks, and R. Schiavilla, Phys. Rev. C 51, 38 (1995).

25. E. Epelbaum, W. Glöckle, and U.G. Meissner, Nucl. Phys. A747, 362 (2005).

26. V.G.J. Stoks et al., Phys. Rev. C 48, 792 (1993).

27. R.A. Arndt et al., SAID, solution of summer 1999 (SM99).

28. D. Alonso and F. Sammarruca, Phys. Rev. C 67, 054301 (2003).

29. Z. H. Li et al., Phys. Rev. C 74, 047304 (2006).

30. L. Coraggio et al., Phys. Rev. C 66, 021303 (2002); ibid. 71, 014307 (2005).

31. P. Navrátil and E. Caurier, Phys. Rev. C 69, 014311 (2004).

32. C. Forssen et al., Phys. Rev. C 71, 044312 (2005).

33. J.P. Vary et al., Eur. Phys. J. A25 s01, 475 (2005).

34. K. Kowalski et al., Phys. Rev. Lett. 92, 132501 (2004).

35. D.J. Dean and M. Hjorth-Jensen, Phys. Rev. C 69, 054320 (2004).

36. M. Wloch et al., J. Phys. G 31, S1291 (2005); Phys. Rev. Lett. 94, 21250 (2005).

37. D.J. Dean et al., Nucl. Phys. A752, 299 (2005).

38. J.R. Gour et al., Phys. Rev. C 74, 024310 (2006).

39. S. Fujii, R. Okamoto, and K. Suzuki, Phys. Rev. C 69, 034328 (2004).

40. K. Ermisch et al., Phys. Rev. C 71, 064004 (2005).

41. H. Witala et al., Phys. Rev. C 73, 044004 (2006).

42. A. Nogga et al., Nucl. Phys. A737, 236 (2004).

43. A. Nogga, P. Navrátil, B.R. Barrett, and J.P. Vary, Phys. Rev. C 73, 064002 (2006).

44. P. Navrátil et al., arXiv:nucl-th/0701038. 